\title{
Statistical Wavelet Features, PCA, MLPNN, SVM and K-NN Based Approach for the Classification of EEG Physiological Signal
}

\author{
Manisha Chandani, Arun Kumar \\ Department of Electronics \& Telecommunication, Bhilai Institute of Technology, Durg, India \\ Email address: \\ chandani.manisha09@gmail.com (M. Chandani), arun.kumar@bitdurg.ac.in (A. Kumar)
}

\section{To cite this article:}

Manisha Chandani, Arun Kumar. Statistical Wavelet Features, PCA, MLPNN, SVM and K-NN Based Approach for the Classification of EEG Physiological Signal. International Journal of Industrial and Manufacturing Systems Engineering. Vol. 2, No. 5, 2017, pp. 57-65. doi: $10.11648 /$ j.ijimse.20170205.12

Received: September 9, 2017; Accepted: September 25, 2017; Published: November 5, 2017

\begin{abstract}
Brain is the most complex organ amongst all the systems in human body. The study of the electrical signals produced by neural activities of human brain is called Electroencephalogram. Electroencephalogram (EEG) is a technique which is used to identify the neurological disorder of brain. Epilepsy is one of the most common neurological disorders of brain. Epilepsy needs to be detected efficiently using required EEG feature extraction such as: mean, standard deviation, median, entropy, kurtosis and skewness etc. The framework of proposed technique is an efficient EEG signal classification approach. The proposed approach is used to classify the EEG signal into two classes: epileptic seizure or not. Extraction of the features by applying Discrete Wavelet Transform (DWT) in order to decompose the EEG signals into sub-bands. These features, extracted from details and approximation coefficients of DWT sub-bands, are used as input to Principal Component Analysis (PCA). The classification is based on reducing the feature dimension using PCA and deriving the Support Vector Machine (SVM), neural network analysis (NNA) and k-nearest neighbour (K-NN). In classification of normal and epileptic, results obtained exhibited an accuracy of $100 \%$ by applying NNA and k-NN. It has been found that the computation time of NNA classifier is lesser than SVM and k-NN to provide 100\% accuracy. So, the detection of an epileptic seizure based on DWT statistical features using NNA classifiers is more suitable in real time for a reliable, automatic epileptic seizure detection system to enhance the patient's care and the quality of life.
\end{abstract}

Keywords: Electroencephalogram (EEGs), Discrete Wavelet Transform (DWT), Principal Component Analysis (PCA), Neural Network Analysis (NNAs), K-Nearest Neighbour (k-NN), Support Vector Machine (SVM), Epileptic, Seizure

\section{Introduction}

Electroencephalogram is the study of the electrical signals produced by brain. Electrical signals generated by the human brain represent the thinking of the mind and the status of the body. The close study of those EEG (EEG) signals is helpful in several analysis areas like detection and classification of event connected potentials, seizure detection and prediction, brain-computer interfacing, Study of mental disorders like psychiatrically disorders, dementedness and sleep signal analysis. For higher understanding of human behaviour the EEG signal waves square measure more divided in five major sub-bands supported the frequency ranges. These bands from low to high frequencies severally square measure known as delta $(\delta)$ (Range $0.5-4 \mathrm{~Hz}$ ), theta $(\theta)$ (Range 4-8 $\mathrm{Hz}$ ), alpha $(\alpha)$ (Range 8-13 Hz), beta $(\beta)$ (Range 13-30 Hz), and gamma $(\gamma)$ (Range 30-45 HZ). [1]. The visual distinction of seizure from common artefacts among associate degree graph measure is predicated on the form and spikiness of the waveforms. A proof with seizure has a tripping and distinguished peaky, whereas the foremost of alternative artefacts square measure non-stationary and every which way formed. However considering the actual fact that the recorded EEG pattern may be a special mapping of signals captured by placement of electrodes onto totally different regions of the scalp, it's very troublesome for person to watch 
and perceive the particular behavior of the brain by just visual review. Therefore there's associate degree everincreasing demand of simply accessible and absolutely automatic convulsion detection system mistreatment EEG signals. To propose options extraction of EEG signals from Discrete wavelet transforms (DWT). Further, support vectormachine (SVM), neural network analysis (NNA) and k-nearest neighbour (K-NN) are employed for classification into two categories that is epileptic and normal. In order to reduce the time and space complexity and to avoid redundancy in the observed features, Principal Component Analysis (PCA) has been applied.

\section{Related Work}

The electrical signals for brain activity were first recorded by the English scientist Richard Caton in 1875. Hans Berger started the study of EEGs from human brain in 1920 [2]. Epilepsy is a Greek word, which means 'to seize or attack'. The very basic concepts of epilepsy can be found in ancient Indian medicine (4500-1500BC) as apasmara, which means "loss of consciousness".

Babylonian tablet in the British Museum in London also gives the detailed knowledge about the epileptic disease and its cure [1]. Kaufman associated the epileptic attacks with abnormal electrical discharges [3]. Most of the epilepsy analysis methods developed in the 20th century were based on the concept of visual inspection of EEG signals by highly skilled electroencephalography's. However, with the advancement in the field of signal processing and pattern recognition, different automatic techniques of epileptic seizure detection have been developed in last two decades [6], [9].

Spectral analysis based feature extraction method provides poor results for EEG classification as the frequency domain information is provided at the cost of time domain information such as the amplitude distribution and EEG pattern. Hence, both time and frequency domain based feature extraction algorithms such as Discrete Wavelet Transform (DWT) are being used in current research [4],[5]. The other advantage of DWT over spectral analysis is its suitability for analysis of non-stationary signals like EEG [6] - [8]. Hilbert-Huang Transformed (HHT) based approach recently published by [9].

\section{Data Selection and Recording}

In this proposed work publicly available data is used which described in [10]. The complete data set consists of five sets (denoted A-E) each containing 100 single-channel EEG segments. These segments were selected and cut out from continuous multi-channel EEG recordings after visual inspection for artefacts, e.g., due to muscle activity or eye movements. Sets A and B consisted of segments taken from surface EEGrecordings that were carried out on five healthy volunteers using a standardised electrode placement scheme.

The characteristics of dataset are explain further and the technique which is used to record the brain signals that is EEG is Bipolar EEG recording.

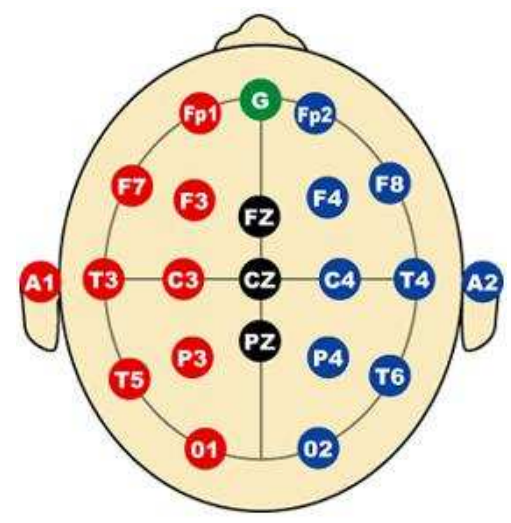

Figure 1. The 10-20 International System of Electrode Placement Images of Normal and Abnormal Cases.

Volunteers were relaxed in associate awake-state with eyes open (A) and eyes closed (B), severally. Sets C, D, and E originated from graph archive of pre-surgical identification. EEGs from 5 patients were hand-picked, all of whom had achieved complete seizure management when surgical operation of one of the hippocampal formations, that was thus properly diagnosed to be the epileptogenic zone. Segments in set D were recorded from among the epileptogenic zone, and people in set $\mathrm{C}$ from the hippocampal formation of the other hemisphere of the brain. Whereas sets C and D contained solely activity measured throughout seizure free intervals, set E solely contained seizure activity. The electrodes were set as shown in figure. 1. Therefore, the electrodes were named as follows:

FP1, FP2, F3, F4, C3, C4, P3, P4, F7, F8, T1, T2, T3, T5, T6, $\mathrm{O} 1, \mathrm{O} 2, \mathrm{~F} 2, \mathrm{P} 2$. Thefrontallobe, temporallobe, parietallobe, centrallobe, and occipital lobe were named F, T, P, C, and Orespectively [11].

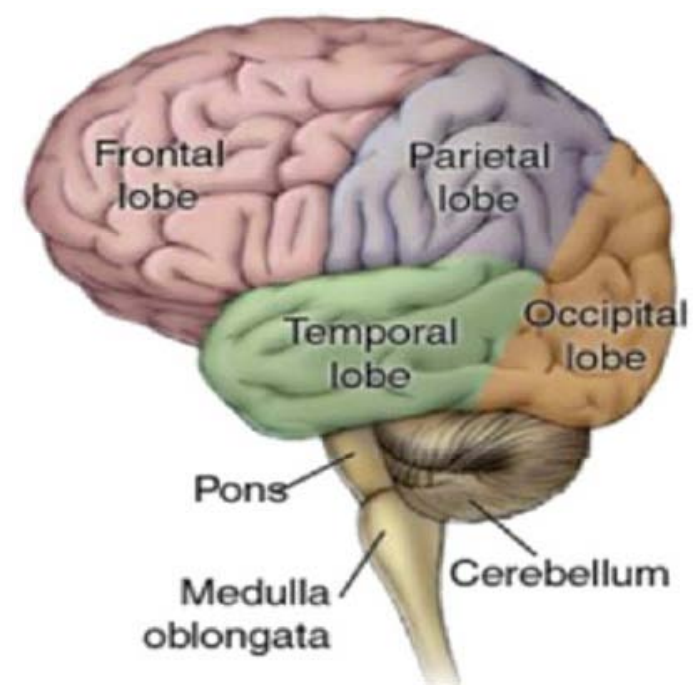

Figure 2. Human Brain Structure.

The Figure 2 describes the anatomy of the brain with different regions [12]. 
The cerebrum is the largest part and is responsible for initiation of movement, coordination of movement, sensing temperature, touch, vision, hearing, judgment, reasoning, problem solving, emotions and learning. Cerebrum is divided into four lobes. They are frontal lobe, occipital lobe, parietal lobe $\&$ temporal lobe. Here segments were selected from all recording sites exhibiting ictal activity. All EEG signals were recorded with the same 128-channel amplifier system, using an average common reference. The data were digitized at 173.61 samples per second using 12 bit resolution. Band pass filter settings were $0.53-40 \mathrm{~Hz}(12 \mathrm{~dB} / \mathrm{oct})$. In this study, two dataset (A and $\mathrm{E}$ ) of the complete dataset is used.
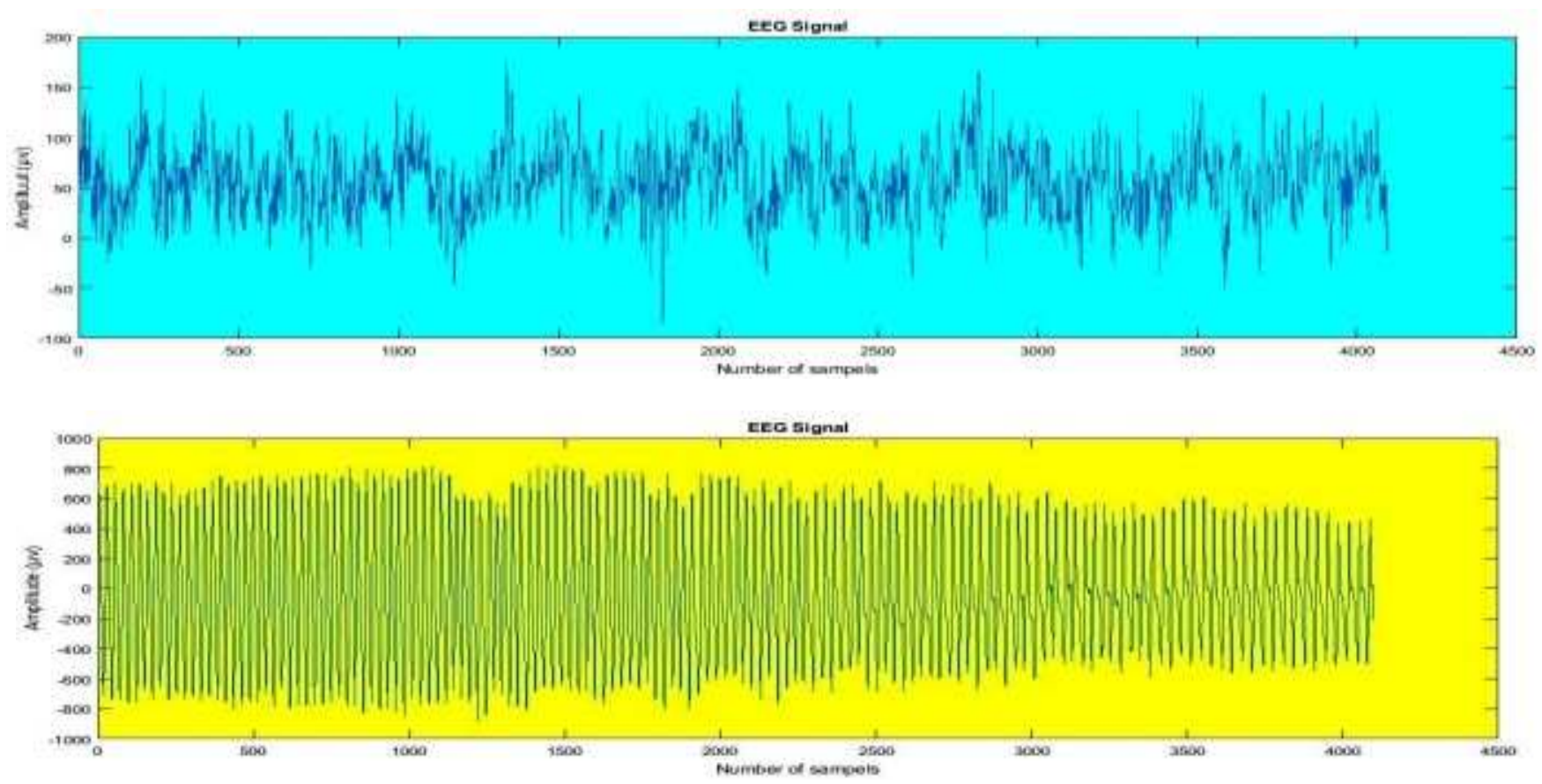

Figure 3. Recorded Healthy Signal and EpilepticSignals.

\section{Methodology}

\subsection{Wavelet Transform for Signal Analysis}

Wavelet transform technique is used to decompose the signals and reconstruct the data accurately. rippling remodel technique could be a appropriate analytical tool for the Non Stationary signals analysis like encephalogram and it had been projected by Jean Morlet a French geologist in 1982 [13] It involves the breaking down of the brain signals into numerous shorter reads of bands as per demand. In distinct wavelet analysis, a multi-resolution description is employed to decompose a given signal $\mathrm{f}(\mathrm{t})$ into progressively finer details supported 2 sets of basic functions [14]. The wavelets and the scaling functions, as follows:

$$
f(t)=\sum_{\mathrm{i}=\mathrm{z}} 2^{\frac{j}{2}} C_{j}(k) \varphi\left(2^{j} t-k\right) \sum_{j=0}^{j-1} \sum_{k=0}^{\infty} 2^{j} d_{j} \psi\left(2^{j} t-k\right)
$$

Where functions. $\varphi(\mathrm{t})$ and $\psi(\mathrm{t})$ are the basic scaling and mother wavelet respectively. Five sub-bands are obtained, namely Delta $(0-4.05 \mathrm{~Hz})$, Theta $(4.05-8.1 \mathrm{~Hz})$, Alpha $(8.1-$ $12.15 \mathrm{~Hz})$, Beta $(12.15-32.5 \mathrm{~Hz})$ and Gamma $(>32.5 \mathrm{~Hz})$ [15].

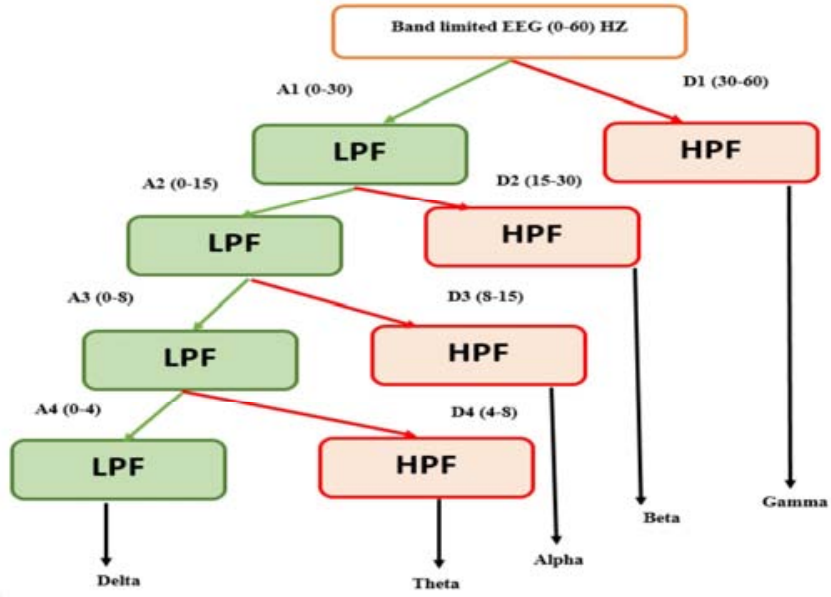

Figure 4. Sub-band decomposition of DWT implementation; the highpass filter, low-pass filter.

The number of decomposition levels is chosen supported the dominant frequency parts of the signal. The amount square measure chosen such those components of the signal that correlates well with the frequencies necessary for classification of the signal square measure maintained within the riffle coefficients. Within the present study, since the encephalogram signals don't have any helpful frequency parts higher than 30 cycles/second, the amount of decomposition levels was chosen to be four. Thus, the encephalogram 
signals were rotten into details D1-D4 and one final approximation, A4. Usually, tests square measure performed with differing kinds of wavelets and also the one, which supplies most potency, is chosen for the actual application.
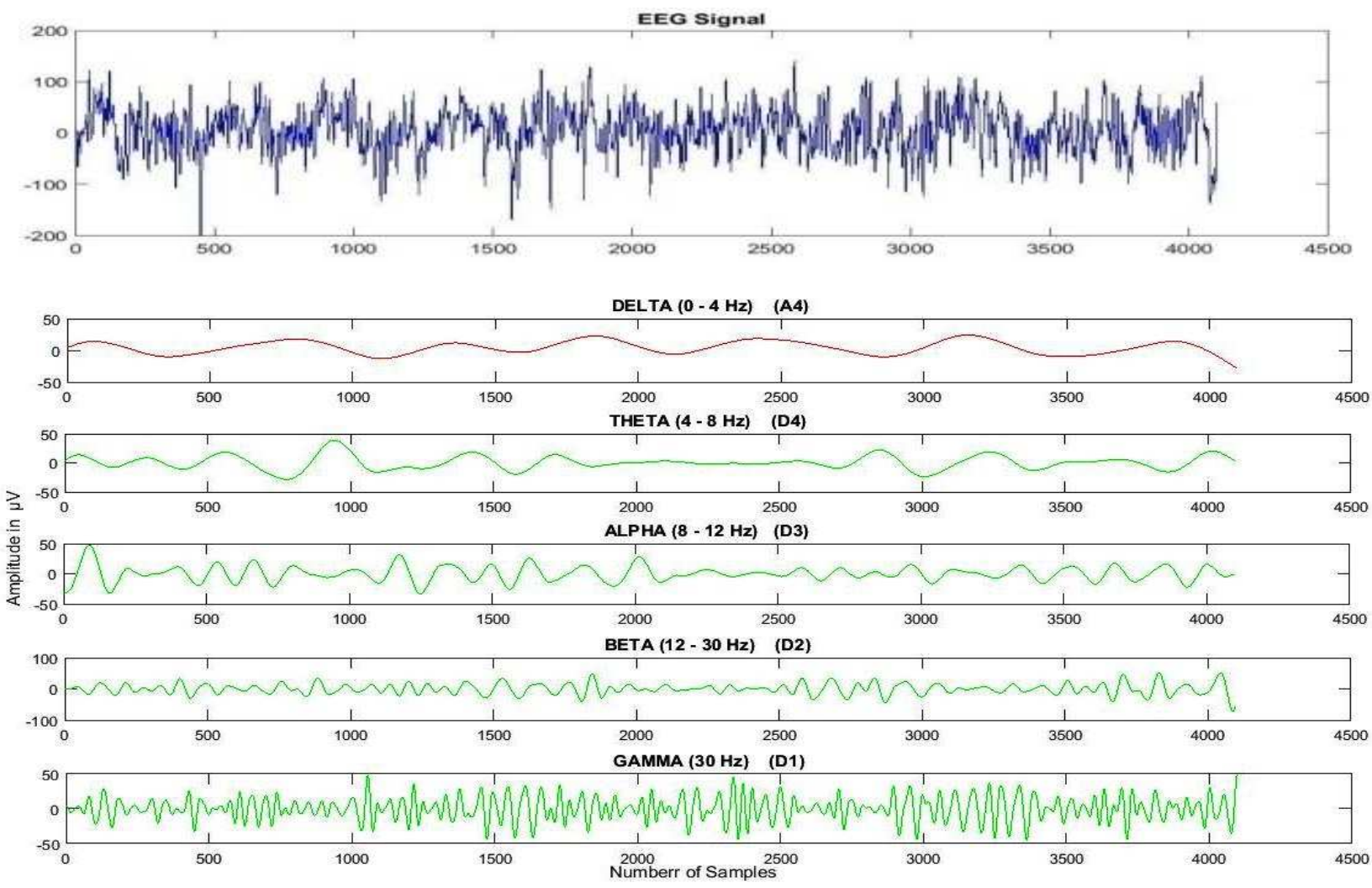

Figure 5. Normal EEG Signal with Delta, Theta, Alpha, Beta and Gamma Subband Decomposition.
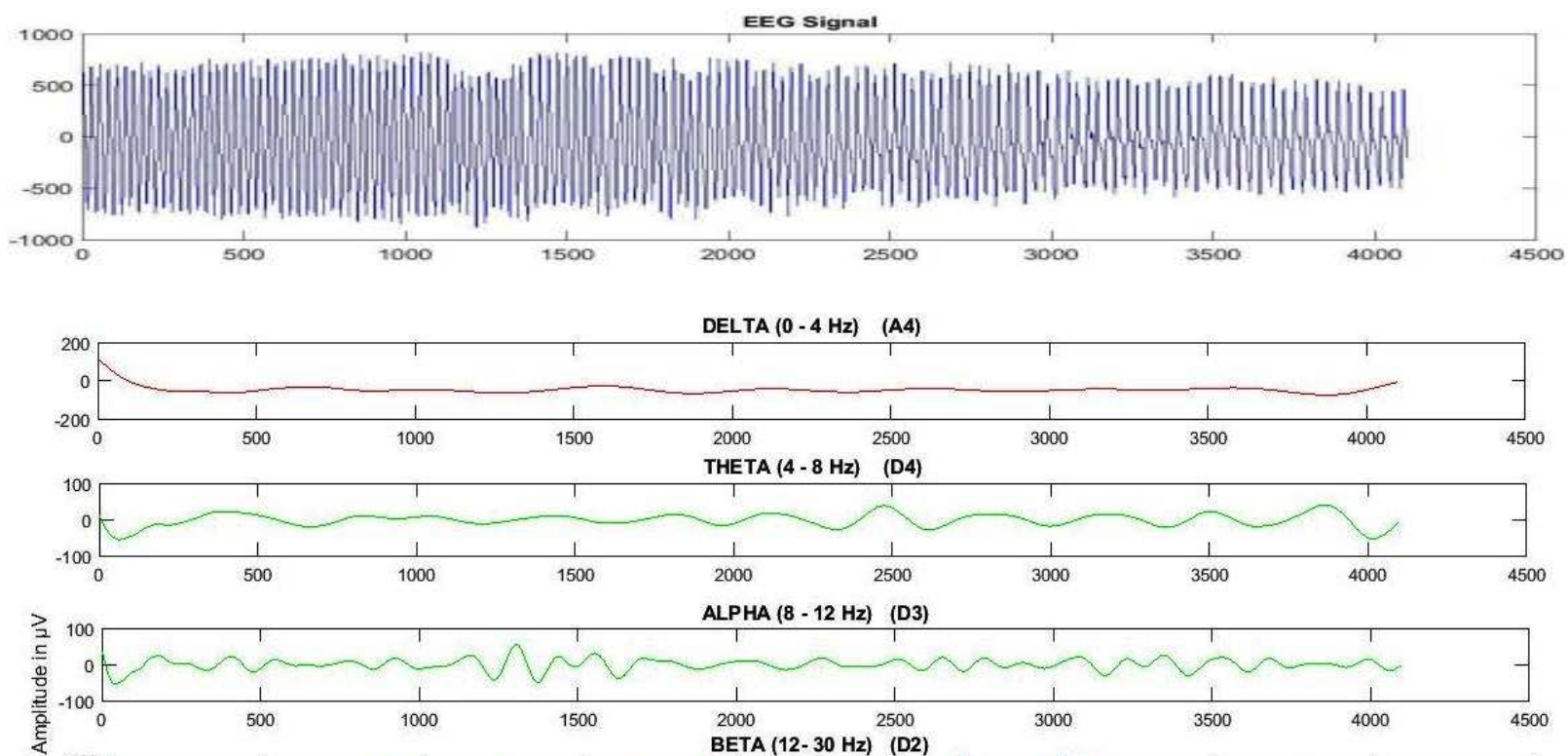

$\underbrace{<}_{0}$

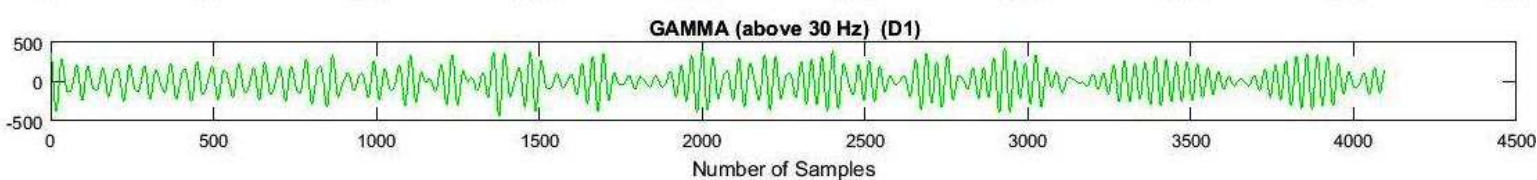

Figure 6. Epileptic EEG Signal with Delta, Theta, Alpha, Beta and Gamma Subband Decomposition. 
The smoothing feature of the Daubechies riffle of order a pair of $(\mathrm{db} 2)$ created it additional applicable to sight changes of encephalogram signals. Hence, the riffle coefficients were computed victimization the $\mathrm{db} 4$ within the present study. The projected methodology was applied on each knowledge set of encephalogram knowledge (Sets A and E).

\subsection{Feature Extraction}

In this work we have chosen the statistical parameters All the extracted features constitute the combined feature index $(\mathrm{CFI})=(\mathrm{F} 1, \mathrm{~F} 2, \mathrm{~F} 3, \mathrm{~F} 4, \mathrm{~F} 5 \ldots . . . \mathrm{Fn})$, which is presented as an input to the ANN network. The features used in evaluating the performance of the proposed scheme are mean, standard deviation, median; entropy, kurtosis and skewness were calculated at each decomposition level starting from 01 to 04 for the normal and epilepsy categories of signals. The mathematical equations are represented as follows:

Mean $\mu$ : Mean are fundamental statistical attributes of a time series the arithmetic mean of a time series is the average expected value of that time series. In some cases, the mean value of a time series can be the operating point or working point of a physical system that generates the time series.

$$
\begin{aligned}
& \mu=\frac{1}{N} \sum_{i=1}^{N} A_{i} \\
& \mathrm{i}=1,2,3, \ldots \ldots, \mathrm{N} .
\end{aligned}
$$

The mean indicated by $\mu$. The Value in the signal $X$, by letting the index, $\mathrm{i}$, run from 0 to 1 . Then finish the calculation by dividing the sum by $\mathrm{N}$. This is identical to the equation: $\mu=\left(X_{0}+X_{1}+X_{2}+\ldots+X_{N-1}\right) / \mathrm{N}$.

Standard Deviation $\sigma$ : The standard deviation is similar to the average deviation except the averaging is done with power instead of amplitude. This is achieved by suaring each of the deviation before taking the average. To finish the square root is taken to compensate for the intial squaring. In equation from the standard deviation is calculation:

$$
\begin{gathered}
\sigma=\sqrt{\frac{1}{N} \sum_{i=1}^{N}\left(x_{i}-\mu\right)^{2}} \\
\mathrm{i}=1,2,3, \ldots \ldots . ., \mathrm{N} .
\end{gathered}
$$

$\mathrm{X}$ is signal with mean $\mu, \mathrm{N}$ is the number of sample and $\sigma$ is standard deviation.

Kurtosis $k$ : The kurtosis are higher-order statistical attributes of a time series. Kurtosis measures the peakedness of the probability density function (PDF) of a time series. A kurtosis value close to three indicates a Gaussian-like peakedness. PDFs with relatively sharp peaks have kurtosis greater than three. PDFs with relatively flat peaks have kurtosis less than three.

$$
k=\frac{E(x-\mu)^{4}}{\sigma^{4}}
$$

Where $\mathrm{X}$ is a signal, $\mu$ is the mean of $x, \sigma$ is the standard deviation of $X$, and $E(t)$ represented the expected value of the quantity $t$ Kurtosis computes a sample version of this population value.

Skewness S: The skewness are higher-order statistical attributes of a time series. Skewness indicates the symmetry of the probability density function (PDF) of the amplitude of a time series. A time series with an equal number of large and small amplitude values has a skewness of zero. A time series with many small values and few large values is positively skewed and the skewness value is positive. A time series with many large values and few small values is negatively skewed and the skewness value is negative.

$$
S=\frac{E(x-\mu)^{4}}{\sigma^{4}}
$$

Where $\mu$ is the mean of signal $x, \sigma$ is the standard deviation of $x$, and $E(t)$ represented the expected value of the quantity $t$ Skewness computes a sample version of this population value.

Entropy E: Entropy is a numerical measure of the randomness of a signal. Entropy can act asa feature and used to analyze psychological time series data such as EEG data. The Entropy can thus be calculated as:

$$
\begin{aligned}
& E(s)=\sum_{i} E\left(s_{i}\right) \\
& \mathrm{i}=1,2,3, \ldots \ldots \ldots, \mathrm{N} .
\end{aligned}
$$

The $\mathrm{E}$ must be the an additive cost function such that $\mathrm{E}(0)$ $=0$. Entropy is the statistical descriptor of the variability within the EEG signal and is a strong feature for epilepsy detection.

Median M: The median is a simple measure of central tendency. To find the median, arrange the observations in order from smallest to largest value. If there are an odd number of observations, the median is the middle value. If there is an even number of observations, the median is the average of the two middle values

$$
\bar{X}=\frac{\sum_{i=1}^{n} X_{i}}{n}
$$

$\mathrm{X}$ refers to the entire set of the numbers. Median are more robust than arithmetic mean and geometric mean if the raw data does not contain significant outliers

\subsection{Classification}

The statistical features derived from DWT are applied to the classifiers. The purpose of the classifier is to identify, epilepsy abnormality in EEG data by linear/non-linear mathematical approach. This proposed work exploit, the classifiers used are Neural Network Analysis and k- nearest neighbour, to identify epileptic seizure EEG data for the individual and combined statistical features derived from DWT with a different combination of data sets A with $\mathrm{E}$. The performance of NNA and k-NN classifiers is assessed with accuracy, sensitivity and specificity for the derived DWT based statistical features to detection the epileptic seizure abnormality. 
Table 1. Nomenclature of Feature Subbands.

\begin{tabular}{llllll}
\hline Sabband Features & Gama & Beta & Alpha & Theta & Delta \\
\hline Mean & M1 & M2 & M3 & M4 & M5 \\
Standard deviation & S1 & S2 & S3 & S4 & S5 \\
Median & Md1 & Md2 & Md3 & Md4 & Md5 \\
Entropy & E1 & E2 & E3 & E4 & E5 \\
Kurtosis & K1 & K2 & K3 & K4 & K5 \\
Skewnes & Y1 & Y2 & Y3 & Y4 & Y5 \\
\hline
\end{tabular}

Using these 30 parameters 80 non-epileptics and 80 epileptic for NNA, 100 non-epileptics and 100 epileptic for SVM and for k-NN 50 non-epileptic and 50 epileptic cases taken from database described before are analyzed.

Classification using NNA: The performance of NNA classifiers is assessed with accuracy, sensitivity and specificity to detection the epileptic seizure abnormality. This task was performed using MATLAB 2015a. In this work Neural network pattern recognition was used for evaluating different states of EEG signals, such as healthy and epileptic states. Structure of Multilayer preceptorn shown in figure 7. In this work the dataset which is used from these data $75 \%$ data from set $\mathrm{A}$ and $75 \%$ data from set $\mathrm{E}$ is used for Training. For testing purpose $25 \%$ data is used. This selected data is used as input to the MLPNN Classifier which is used in this work.

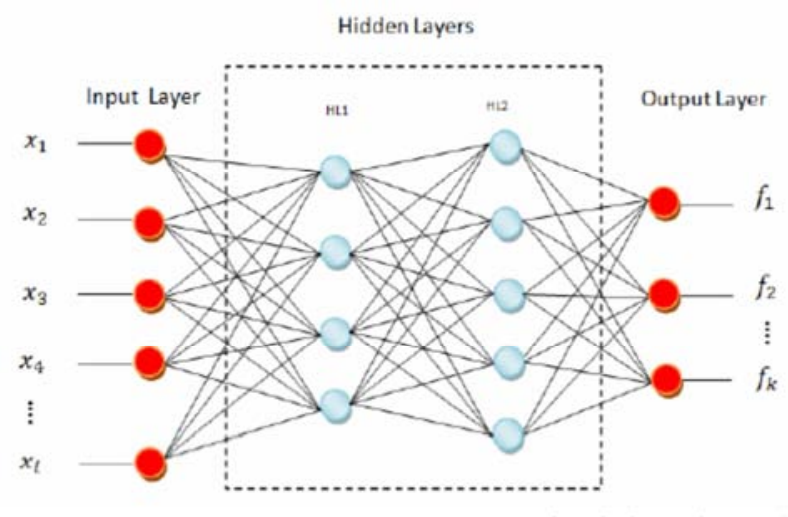

Figure 7. Multilayer Perceptron.

The number of neurons in the input layer symbolizes the number of features presented to the network, followed by hidden layer with neurons which transforms the input into nonlinear combinations and passes the signals to the output layer [16]. In our study, the number of neurons in the input layer is thirteen corresponding to CFI and the number of neurons in the output layer is three to classify three different classes.

The most challenging task is to select the number of neurons in hidden layer. In this framework varied the numbers of hidden nodes to find out the architecture giving the better performance with high accuracy. This work adapted a method by systematically enumerating all combinations of feature vectors and checking all different size CFIs for the optimality of the features.

Classification using SVM: The idea of SVM is originated from the idea of controlling the generalizing capabilities of machines for automation. The performance of a classifier must be generalized, i.e. it should perform well when it is applied for the data outside the training set. The notion of maximizing the margin between the support vectors is at the heart of the SVM classifier, in order to perform more accurately on unknown data [17], [18] Consider the hyper-plane in (8):

$$
W^{T} x+W_{0}=0
$$

The margin is the Euclidian distance $1 /\|w\|$ between the two parallel hyper-planes (support vectors) described $\mathrm{i}$ n (9):

$$
x+W_{0}=1, \text { and } W^{T} x+W_{0}=-1
$$

Let $x_{i}$ are training points, with respective classes $y_{i} \in\{-$ $1,1\}, \mathrm{i}=1,2, \ldots, \mathrm{N}$ for a 2 class classification problem. The task is to optimize for minimum training error and maximum separating margin between hyper-planes of (9). SVM classifier solves this task by solving the optimization problem of (10):

$$
\begin{aligned}
& \text { Minimize } \mathrm{L}\left(W, W_{0}, \xi\right)=\frac{1}{2}\|W\|^{2}+C \sum_{i=1}^{N} \xi_{i} \\
& \text { Subjected } W^{T} x+W_{0} \geq 1-\xi_{i}, \text { if } y_{i} \in 1 \\
& \text { And } W^{T} x+W_{0} \leq 1+\xi_{i}, \text { if } y_{i} \in-1
\end{aligned}
$$

$$
\text { And } \xi_{i} \geq 0
$$

For the present work, involving two class classification (Epileptic seizure or not), firstly learn the classifier equation (similar to (8)) by solving the optimization problem of (10) with constrains of (11), using half of the feature vectors from Matrixes training data. It is found that the entropy and kurtosis from all the five sub-bands are optimum for classification of EEG signals and it gives high performance accuracy.

Classification using $k$-NN: The k-Nearest Neighbour (KNN) classifier is used for detection of epileptic subjects k$\mathrm{NN}$ is a supervised learning algorithm. The classification of this algorithm is based on using a reference data set containing both the input and the target members and then it compares the unknown input members to that reference data set.

Assignment of the class is done by determining the distance of the unknown to the $\mathrm{k}$ nearest neighbors by obtaining a majority vote from them. From table 130 parameters 50 non-epileptic and 50 epileptic cases taken from database described before are analyzed. For improving classification performance, different number of feature was used to obtain the different results with the highest accuracy. In the first step, 50 mean values, in the second step 50 Entropy \& in the third step 50 skewness values for each segment were extracted as features. For improving classification performance, the features were reduced to 30 feature by applying minimal redundancy- maximal relevance criterion (MRMR) [19].

Minimum redundancy feature selection is an algorithm frequently used in a method to accurately identify 
characteristics of genes and phenotypes and narrow down their relevance and is usually describe in its pairing with relevant feature selection as Minimum Redundancy Maximum Relevance (MRMR).

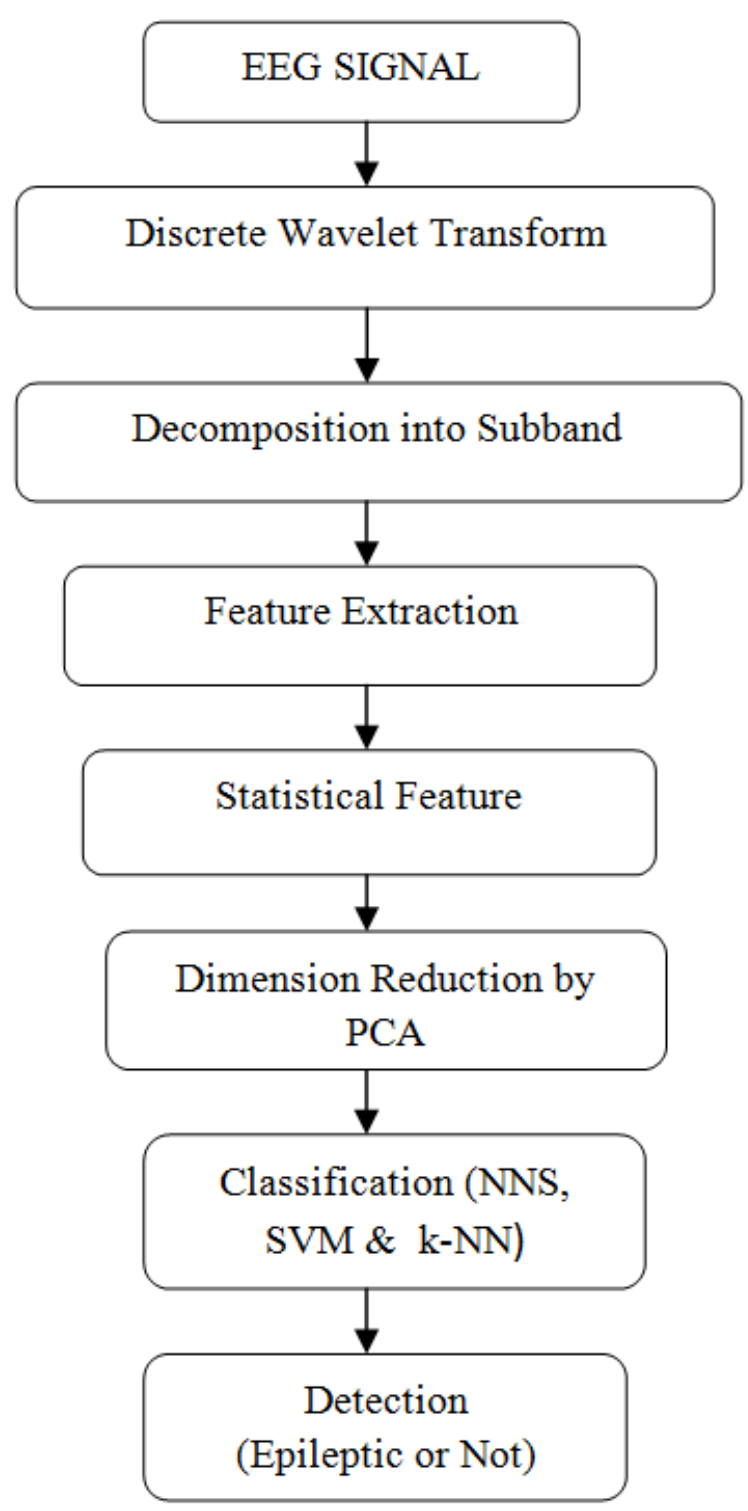

Figure 8. General Block Diagram for Seizure Classification.

\section{Experimental Result}

In this process classification is done for ictal state $(\mathrm{E})$ and other healthy state (A) and in order to measure the performance of the classifier, Sensitivity (TPR) and Specificity (TNR) were calculated and further total Accuracy will calculated with the help of all above.

Sensitivity (TPR): The sensitivity of a test is its ability to determine the patient cases correctly. To estimate it should calculate the proportion of true positive in patient cases [20].

Specificity (TNR): The specificity of a test is its ability to determine the healthy cases correctly. To estimate it should calculate the proportion of true negative in healthy cases [20].
Accuracy: The accuracy of a test is its ability to differentiate the patient and healthy cases correctly. To estimate the accuracy of a test should calculate the proportion of true positive and true negative in all evaluated cases [20].

Equation given below describes the formula used for calculating Sensitivity and Specificity using:

TPR $=$ True Positive Ratio

TNR $=$ True Negative Ratio

$\mathrm{TP}=$ True Positive

$\mathrm{TN}=$ True negative

$\mathrm{FP}=$ False Positive

$\mathrm{FP}=$ False Positive

$\mathrm{FN}=$ False Negative.

Mathematically, this can be stated as:

$$
\begin{aligned}
& \text { Sensitivity }=\mathrm{TPR}=\frac{\mathrm{TN}}{\mathrm{TP}+\mathrm{FN}} \times 100 \% \\
& \text { Specificity }=\mathrm{TNR}=\frac{\mathrm{TP}}{\mathrm{TN}+\mathrm{FP}} \times 100 \% \\
& \text { Accuracy }=\frac{\mathrm{TP}+\mathrm{TN}}{\mathrm{TP}+\mathrm{FP}+\mathrm{TN}+\mathrm{FN}} \times 100 \%
\end{aligned}
$$

Table 2. General Confusion Matrix.

\begin{tabular}{cccc}
\hline \multicolumn{4}{c}{ Predicted Class } \\
\hline \multirow{2}{*}{ Actual } & Class & Yes & No \\
Class & Yes & TP & FN \\
& No & FP & TN \\
\hline
\end{tabular}

Performance of NNA Classifier

Table 3. Confusion Matrix for Normal and Epileptic Detection.

\begin{tabular}{clcc}
\hline \multicolumn{4}{c}{ Predicted Class } \\
\hline Normal with Eye & Class & Epileptic & Normal \\
open and Epileptic & Yes & 80 & 0 \\
classification & No & 0 & 80 \\
\hline
\end{tabular}

The values obtained for performance measuring Parameters is shown in table 4

Table 4. Performance Table of NNA Classifier.

\begin{tabular}{cccc}
\hline Normal with Eye open and & Accuracy & Sensitivity & Specificity \\
\cline { 2 - 4 } Epileptic classification & $100 \%$ & $100 \%$ & $100 \%$ \\
\hline
\end{tabular}

Performance of SVM Classifier

Table 5. Confusion Matrix for Normal and Epileptic Detection.

\begin{tabular}{cccc}
\hline \multicolumn{4}{c}{ Predicted Class } \\
\hline \multirow{2}{*}{ Normal with Eye open and } & Class & Epileptic & Normal \\
Epileptic classification & Yes & 98 & 2 \\
& No & 100 & 0 \\
\hline
\end{tabular}

The values obtained for performance measuring Parameters is shown in table 6

Table 6. Performance of SVM Classifier.

\begin{tabular}{cccc}
\hline Normal with Eye open and & Accuracy & Sensitivity & Specificity \\
\cline { 2 - 4 } Epileptic classification & $99 \%$ & $98 \%$ & $100 \%$ \\
\hline
\end{tabular}

Performance of k-NN Classifier 
Table 7. Confusion Matrix for Normal and Epileptic Detection.

\begin{tabular}{cccc}
\hline \multirow{2}{*}{ Normal with Eye open and } & Class & Epileptic & Normal \\
\cline { 2 - 4 } Epileptic classification & Yes & 50 & 0 \\
& No & 0 & 50 \\
\hline
\end{tabular}

The values obtained fo performance measuring Parameters is shown in table 8

Table 8. Performance of $k-N N$ Classifier.

\begin{tabular}{cccc}
\hline Normal with Eye open and & Accuracy & Sensitivity & Specificity \\
\cline { 2 - 4 } Epileptic classification & $100 \%$ & $100 \%$ & $100 \%$ \\
\hline
\end{tabular}

\section{Conclusion}

An expert model was developed for detection of epilepsy on the background of EEG by using discrete wavelet transform and SVM, MLPNN and k-NN classifiers. The feature like Mean, median, entropy, standard deviation, kurtosis, skewness was extracted from the EEG signal. The results have been shown that the proposed technique could attain a higher accuracy using SVM, MLPNN and k-NN both the classifier for normal eyes open and epileptic seizure EEG data sets for all individual and combined statistical features derived from DWT for the detection of an epileptic seizure. The study of the proposed technique is evident from the other pattern recognition approaches considered by the researchers for different combination of data set $\mathrm{A}$ and $\mathrm{D}$ and this work confirm that the NNA classifier achieves high accuracy with less computation time over SVM and k-NN.

\section{Acknowledgements}

The authors are very much thankful to Dr. R. G. Andrzejak of University of Bonn, Germany, for providing permission to use the EEG data available in the public domain [10]. I owe a debt of gratitude to my project guide Dr. Arun Kumar Associate Professor, Department of Electronics \&Telecommunication Engineering, for his right orientation, invaluable knowledge. His critical judgment and review shaped this paper to add with a quality manuscript.

\section{References}

[1] Saeid Sanei and J. A. Chambers EEG Signal Processing. John Wiley \& Sons, 2007.

[2] A. Massimo. "In Memoriam Pierre Gloor 1923-2003): an appreciation”. Epilepsia, vol.-45(7), page-882, 2004.

[3] M. A. B. Brazier. "A History of the Electrical Activity of the Brain". The First Half-Century, Macmillan, New York, 1961.

[4] M. D. Alessandro, R. Esteller\& G. Vachtsevanos. A. Hinson, A. Echauz, and B. Litt. "Epileptic seizure prediction using hybrid feature selection over multiple intracranial EEG electrode contacts: A report of four patients". IEEE Transactions on Biomedical Engineering. vol. 50 (5), pp.-603-615, 2003.
[5] B. P. Marchant. "Time-frequency analysis for biosystem engineering". Biosystems Engineering. vol.-85 (3), pp.261-281, 2003.

[6] A. Subasi. "EEG signal classification using wavelet function extraction and a mixture of expert model', Expert System with Application, 32, 1084-1093, 2007.

[7] A. Subasi, and M. Ismail Gursoy. "EEG signal classification using PCA, ICA, LDA and support vector machines". Expert Systems with Applications. vol. 37, pp.8659-8666, 2010.

[8] A. Subas. "Epileptic seizure detection using dynamic wavelet network". Expert Systems with Applications.. vol.29, pp.-343-355, 2005.

[9] K. Fu, J. Qu, Y. Chai, \& Y. Dong. "Classification of seizure based on the time-frequency image of EEG signals using HHT and SVM". Biomedical Signal Processingand Control. vol.-13, pp.-15-22, 2014.

[10] R. G. Andrezejak, K. Lehnertz \& F. Morman. Indication of nonlinear deterministic and finite-dimensional structures in time series of brain electrical activity: dependence on recording region and brain state. Phys. Rev. Ed-64 (6) 061907, 2001.

[11] Durka P. J. Adaptive time-frequency parametrization of epileptic spikes. Physical Review E; 69: 051914, 2004.

[12] Kumari Pinki \& Abhishek Vaish. Brainwave based user identification system: A pilot study in robotics environment. Robotics and Autonomous Systems 65, pp. 15-23, 2015.

[13] A. Subasi. "EEG signal classification using wavelet function extraction and a mixture of expert model", Expert System with Application, 32, pp.1084-1093, 2007.

[14] C. S. Burrus, R. A. Gopinath, \& H. Guo (1998). Introduction to wavelets and wavelet transforms: A primer. Prentice-Hall, Upper Saddle River, NJ.

[15] Mandeep Singh \& Sunpreet Kaur. Epilepsy, "Frequency Band Separation for Epilepsy Detection Using EEG”, International Journal of Information Technology \& Knowledge Management, Vol 6, No.1, 2012.

[16] Claude Roberta, Jean-Franc, ois Gaudyb \& Aime' Limogea. "Electroencephalogram processing using neural networks", Clinical Neurophysiology 113, pp.694-701, 2002.

[17] S. Theodoridis \& K Koutroumbas. Pattern Recognition. $4^{\text {th }}$ Ed Elsevier - Academic Press, 2009.

[18] P. S. Sastry. "An introduction to Support Vector Machines". Chapter in J. C. Misra (Ed), computing and information sciences: Recent Trends. Narosa Publishing House, New Delhi, 2003.

[19] H. Peng, F. Long, \& C. Ding. "Feature selection based on mutual information: criteria of max-dependency, maxrelevance, and min-redundancy". IEEE Transactions on Pattern Analysis and Machine Intelligence, vol. 27, No. 8, pp. 1226- 1238, 2005.

[20] Alireza Baratloo, Mostafa Hosseini, Ahmmed Negida \& Gehad El Ashal. "Simple Definition and Calculation of Accuracy, Sensitivity and Specificity". Volume 4 No. 2, pp. 48-49, 2015. 
Approach for the Classification of EEG Physiological Signal

[21] Semwal, Vijay Bhaskar, Manish Raj, and Gora Chand Nandi. "Biometric gait identification based on a multilayer perceptron." Robotics and Autonomous Systems 65 pp. 65-75, 2015.

[22] Khan Y. U, Farooq O \& Sharma P. "Automatic detection of seizure onset in pediatric EEG". International Joural of Embeded Systems and Applications. Vol. 2 pp. 81-89, 2012.
[23] Meier R, Dittrich H, Schulze-Bonhage A \& Aertsen A. "Detecting epileptic seizures in long-term human EEG: a new approach to automatic online and real-time detection and classification of polymorphic seizure patterns". Journal of Clinical Neurophysiology Vol25, pp. 119-131, 2008.

[24] Stein A. G, Eder H. G, Blum D. E, Drachev A \& Fisher R. S. "An automated drug delivery system for focal epilepsy". Epilepsy research Vol.39, pp. 103-114, 2000. 